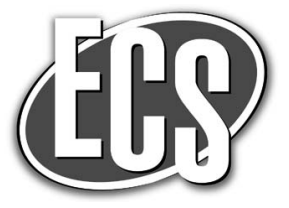

\title{
Simulation and Experiment on Solid Electrolyte Interphase (SEI) Morphology Evolution and Lithium-Ion Diffusion
}

\author{
Pengjian Guan, Lin Liu, ${ }^{*, z}$ and Xianke Lin \\ Department of Mechanical Engineering, The University of Kansas, Lawrence, Kansas 66045, USA
}

\begin{abstract}
In this study, a phase-field model is developed to simulate the microstructure morphology evolution that occurs during solid electrolyte interphase (SEI) growth. Compared with other simulation methodologies, the phase-field method has been widely applied in the solidification modeling that has great relevance to SEI formation. The developed model can simulate SEI structure and morphology evolution, and can predict SEI thickness growth rate. X-ray photoelectron spectroscopy (XPS) experiments are performed to confirm the major SEI species as $\mathrm{LiF}, \mathrm{Li}_{2} \mathrm{O}, \mathrm{ROLi}$, and $\mathrm{ROCO}_{2} \mathrm{Li}$. Transmission electron microscopy (TEM) experiment is performed to present the SEI layer structures. The experiments reduce the complexity of the model development and provide validation to some extent. Fick's law and mass balance are applied to investigate lithium-ion concentration distributions and diffusion coefficients in different types of SEI layers predicted by the phase-field simulations. Simulation results show that lithium-ion diffusion coefficients between $298 \mathrm{~K}$ and $318 \mathrm{~K}$ are $1.340-7.328\left(10^{-16}\right) \mathrm{cm}^{2} / \mathrm{s}, 1.734-3.405\left(10^{-12}\right) \mathrm{cm}^{2} / \mathrm{s}$, and $2.611-2.389\left(10^{-15}\right) \mathrm{cm}^{2} / \mathrm{s} \mathrm{in} \mathrm{the} \mathrm{compact,}$ porous, and multilayered structures of SEI layer, respectively. The resistances between $298 \mathrm{~K}$ and $318 \mathrm{~K}$ are $0.740-1.693 \Omega \cdot \mathrm{cm}^{2}$, 2.827-5.517 $\Omega \cdot \mathrm{cm}^{2}$, and 3.726-5.839 $\Omega \cdot \mathrm{cm}^{2}$ in the compact, porous, and multilayered structures of SEI layer, respectively.

(C) The Author(s) 2015. Published by ECS. This is an open access article distributed under the terms of the Creative Commons Attribution 4.0 License (CC BY, http://creativecommons.org/licenses/by/4.0/), which permits unrestricted reuse of the work in any medium, provided the original work is properly cited. [DOI: 10.1149/2.0521509jes] All rights reserved.
\end{abstract}

Manuscript submitted April 20, 2015; revised manuscript received May 22, 2015. Published June 23, 2015. This was Paper 32 presented at the Orlando, Florida, Meeting of the Society, May 11-15, 2014.

Lithium-ion batteries (LIBs) are widely used in many applications, such as cell phones, electric vehicles (EVs), and other energy storage modules. However, LIBs suffer from severe performance degradation due to undesired chemical reactions, ${ }^{1}$ ageing, ${ }^{2,3}$ corrosion, ${ }^{4-6}$ compromised structural integrity, ${ }^{7,8}$ and thermal runaway. ${ }^{9-11}$ The degradation occurs during both calendar and cycling lifespans, and reduces the longevity of LIBs. Recently, much attention has been focused on LIB material decomposition, ${ }^{12}$ e.g., the formation and growth of new components ${ }^{13-19}$ due to undesired side reactions. ${ }^{1,20}$ The main degradation mechanisms in LIBs vary with different active materials, ${ }^{2}$ however, it is well known that a carbonaceous lithium-intercalation electrode in contact with electrolyte solution becomes covered by a passivation layer called a solid electrolyte interphase (SEI). While SEI can prevent the exfoliation of graphite materials and inhibit further electrolyte decomposition. ${ }^{21}$ SEI layer growth can also cause battery capacity fade and increase cell internal resistance. ${ }^{17,22-26}$ Therefore, the study of SEI plays a key role in battery degradation and other related performance improvement research. Many studies have been published in SEI computational and experimental studies, including but not limited to. ${ }^{27-30}$

Many researchers have investigated SEI in LIBs in terms of structure, ${ }^{7,8,29,31-33}$ formation and composition, ${ }^{20,22,27}$ and thickness growth prediction and measurement. ${ }^{15,16,34,35} \mathrm{SEI}$ is believed to have a multilayered structure: a compact layer of inorganic components (e.g., $\mathrm{LiF}, \mathrm{Li}_{2} \mathrm{O}$ ) close to graphite electrode followed by a porous organic layer (e.g., $\mathrm{ROLi}, \mathrm{ROCO}_{2} \mathrm{Li}$ ) close to the electrolyte solution phase..$^{22,29,31-33}$ The composition of SEI depends on the electrode materials and electrolyte composition. ${ }^{27}$ Broussely et al. investigated the mechanism of lithium loss in LIBs during storage, and their developed diffusion-limited SEI growth model revealed that the rate of lithium loss is proportional to the SEI electronic conductance. ${ }^{28}$ The work by Borodin et al. showed that the nature of the electrolyte has a fundamental impact on the formation and composition of SEI. ${ }^{27}$ Kim et al. carried out simulations to study the effect of the electrolyte on the composition of SEI. They found that $\mathrm{Li}_{2} \mathrm{CO}_{3}$ and $\mathrm{Li}_{2} \mathrm{O}$ are the primary inorganic components of SEI above a lithium metal anode when Ethylene Carbonate (EC) is used as the electrolyte. ${ }^{29}$ Liu et al. developed a thermal-electrochemical model to study SEI growth and electrochemical impedance spectroscopy. ${ }^{36}$ Newman et al. developed a macroscopic model to simulate the growth of SEI layer and the

*Electrochemical Society Active Member.

${ }^{\mathrm{z}}$ E-mail: linliu@ku.edu transport of lithium ions in SEI. ${ }^{37}$ They also investigated the layer growth rate, cell resistance, and increase in irreversible capacity loss during the formation and growth of SEI layer. ${ }^{37}$ In addition, the model developed by Liu et al. showed the impacts of the competing mechanisms of diffusivity and reaction kinetics on SEI layer growth and cell capacity fade considering the effect of temperatures. ${ }^{38}$

However, evolutionary processes of SEI layer formation and growth are still not fully understood, especially the microstructure morphology evolution of SEI and lithium-ion diffusion inside the SEI layer. Yan's phenomenological model elaborated on the details of SEI formation and evolution during the first electrochemical intercalation of lithium into graphite. ${ }^{31}$ Peled's mosaic block model showed a hypothetical description of the SEI morphology. ${ }^{33}$ Similar to our previous work, ${ }^{39}$ the formation and morphology evolution of SEI are simplified as a solidification process. Initially the electrolyte solution becomes unstable, and reduction reactions happen with electrons from the electrode to form SEI species (solid phase) in the electrolyte solution (liquid phase) that may undergo decomposition. ${ }^{28}$ SEI species accumulate and form a passivation layer with multilayered structure (i.e., compact and porous layers) that hinders direct contact between the electrolyte solution and electrode surface. Since the porous SEI layer has lower electronic conductivity than the compact layer, it will stop growing when the electrolyte solution molecules can no longer receive electrons from the electrode to be further reduced. Consequently, SEI growth rate reduces and SEI layer stabilizes. Although the dissolution of SEI may occur, this work has not considered the dissolution mechanism due to computational complexity of phase-field simulation. The SEI growth process shares many similarities with solidification. Ode et al. demonstrated that the phase-field method can identify solidification problems. ${ }^{40}$ Chen et al. reviewed the development of phase-field simulation for solidification, ${ }^{41}$ and Deng et al. recently used phase-field simulation to investigate the formation of SEI. ${ }^{42}$ The aforementioned studies provide the physical justification for assuming the solidification to be governed by the free energy gradient. ${ }^{42}$ In this study, phase-field method is adopted to study SEI layer morphology evolution. SEI layer morphology evolution and growth involves many boundaries of reaction products and their dynamic interfaces. Instead of directly tracking the interface between two phases, the diffusive interface between the electrolyte solution and SEI species is assumed to be governed by a dimensionless phasefield variable, $\varphi .^{42-49}$ The detailed chemistry of SEI species formation and complicated mechanisms of electron/charge transportation have not been considered in this study. However, experiments were carried out to confirm major the SEI species $i$ with given concentration $\mathrm{Ci}$ 
in electrolyte solution. Then, we focus on the SEI layer morphology evolution and growth during the minimization of total free energy density of each SEI species. Electric potential and elastic energy are not included so as to reduce computational complexity. A diffusion model based on Fick's law and mass balance is developed to investigate the lithium-ion diffusion in different types of SEI layers predicted by the phase-field simualtions.

\section{Methodology}

The phase-field variable $(\varphi)$ corresponds to the solid/liquid volume fraction $\left(V_{f}\right)$ in the SEI/electrolyte interface.

$$
\varphi\left(C_{i}, t\right)=2 V_{f}-1
$$

The volume fraction $\left(V_{f}\right)$ ranges from 0 to 1 , where $V_{f}=0,1$ represent liquid and solid phase, respectively. $C_{i}$ is the concentration of SEI species $i$, and $\mathrm{t}$ is the time during the SEI formation. Therefore, $\varphi\left(C_{i}, t\right)=1$ signifies solid SEI phase, $\varphi\left(C_{i}, t\right)=-1$ corresponds to liquid electrolyte phase, and $-1<\varphi<1$ represents the SEI/electrolyte interface. As shown in Figure 1, a two-phase system in a localized region is described by three domains: SEI phase, electrolyte phase, and a diffusive interface region. The thickness of the interface region is represented by $\varepsilon$. There is no pure liquid (electrolyte) or pure solid (SEI species) inside the diffusive interface region. The evolution of $\varphi$ is governed by the Cahn-Hilliard equation. ${ }^{47,50,51}$ The phase-field model development is based on energy conservation, mass conservation, momentum balance, and phase separation at the interface, which can be referred to in detail in our previous work. ${ }^{39,52}$

The key aspect of SEI species formation and evolution is the minimization of total free energy of the two-phase system. The total free energy comes from two components: mixing energy of the $\mathrm{SEI} /$ electrolyte interface and elastic energy. Since elastic energy is not considered in this work, the Ginzburg-Landau form is applied for the total free energy density: ${ }^{51}$

$$
f_{\text {tot }}=\frac{1}{2} \lambda|\nabla \varphi|^{2}+f_{0}(\varphi)^{2}
$$

where $f_{\text {tot }}$ is the total free energy density, $\lambda$ is the free energy gradient, and $f_{0}$ is a function of the bulk energy density. The choice of bulk energy density function can have a significant effect on the physical behavior of the interface. In this work, a double-well function for $f_{0}$ is applied.

$$
f_{0}=\frac{\lambda}{4 \varepsilon^{2}}\left(\varphi^{2}-1\right)^{2}
$$

The physical justification of $f_{0}$ comes from the separation of phases into domains of pure components $(\varphi= \pm 1) .{ }^{53}$ The double-well function is commonly used when the phase-field model is applied for interface tracking purposes, and represents an approximation of the Van der Waals Equation of State near the critical radius, $\mathrm{R}_{\mathrm{i}}{ }^{*}$. However,

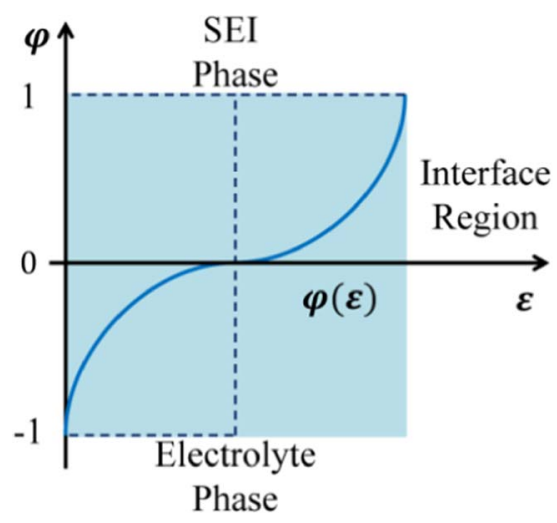

Figure 1. Phase-field variable $\varphi(\varepsilon)$ represents a diffusive interface. this approximation may cause the spontaneous solid species shrinkage phenomenon. ${ }^{54}$ In a solid/liquid two-phase system, the total free energy density can be divided into surface free energy density $\left(f_{\mathrm{s}}\right)$ and volume free energy density $\left(f_{\mathrm{v}}\right) . f_{\text {tot }}$ reaches its maximum value when a SEI species $i$ has the critical radius $\mathrm{R}_{\mathrm{i}}{ }^{*}$. If $\mathrm{R}_{\mathrm{i}}$ is larger than $\mathrm{R}_{\mathrm{i}}{ }^{*}$, volume free energy density is dominant, and the SEI species $i$ may tend to grow since the total free energy density is increasing. On the other hand, if $\mathrm{R}_{\mathrm{i}}$ is smaller than $\mathrm{R}_{\mathrm{i}}{ }^{*}$, surface free energy density is dominant, and the SEI species $i$ may tend to shrink and eventually dissolve into the electrolyte solution since the total free energy density is decreasing. Yue et al. theoretically investigated the calculation of the critical radius. Their results indicated that changes in size of solid species are proportional to the thickness of interface $\varepsilon .{ }^{54}$ As $\varepsilon \rightarrow 0$, the ratio $\lambda / \varepsilon$ obtains the surface tension coefficient, $\sigma_{s e}$, as shown in Figure 2.

$$
\sigma_{s e}=\frac{2 \sqrt{2}}{3} \frac{\lambda}{\varepsilon}
$$

In this work, it is assumed that the SEI layer is formed by different precipitated solid species in the electrolyte. As a result, the SEI/electrolyte interface tends to keep a spherical configuration to minimize the surface free energy density. ${ }^{31}$ Figure 2 shows a schematic of contact angle and surface tension coefficients. Young's equation is applied to connect the contact angle $(\theta)$ with the SEI/electrolyte interfacial tension coefficient $\left(\sigma_{s e}\right)$, the SEI/graphite surface tension coefficient $\left(\sigma_{s g}\right)$, and the electrolyte/graphite surface tension coefficient $\left(\sigma_{e g}\right)$.

$$
\cos (\theta)=\frac{\sigma_{s g}-\sigma_{e g}}{\sigma_{s e}}
$$

Thus, the surface free energy density can be represented as:

$$
f_{s}=\sqrt[3]{4} \pi R_{i}^{2}\left(2 \sigma_{s e}+\sigma_{s g}\right)
$$

where $\mathrm{R}_{\mathrm{i}}$ is the initial radius of the SEI species $i$.

The phase-field variable $(\varphi)$ is a conserved property since the concentration field is a conserved property during long-range diffusion. Therefore, the evolution of $\varphi$ can be governed by the Cahn-Hilliard equation:

$$
\frac{\partial \varphi}{\partial t}+\mathbf{v} \cdot \nabla \varphi=\gamma \nabla^{2} \omega
$$

where $\mathbf{v}$ is the flow velocity for advection, $\omega$ is the chemical potential coefficient, and $\gamma$ is the mobility of the interface with a range of $0 \leq \gamma \leq 1 . \gamma$ is used to determine the time scale of Cahn-Hilliard diffusion and control the minimization of the total free energy density. Here, the chemical potential coefficient of SEI species is written as:

$$
\begin{gathered}
\omega=\lambda \frac{\delta\left(f_{\text {tot }}\right)}{\delta \varphi} \\
\omega=\frac{\lambda}{\varepsilon^{2}}\left[-\varepsilon^{2} \cdot \nabla^{2} \varphi+\varphi\left(\varphi^{2}-1\right)\right]
\end{gathered}
$$

The Cahn-Hilliard equation forces $\varphi$ to take a value of 1 or -1 , and can be represented by two second-order PDEs:

$$
\frac{\partial \varphi}{\partial t}+\mathbf{v} \cdot \nabla \varphi=\frac{\gamma \lambda}{\varepsilon^{2}} \nabla^{2} \varphi
$$

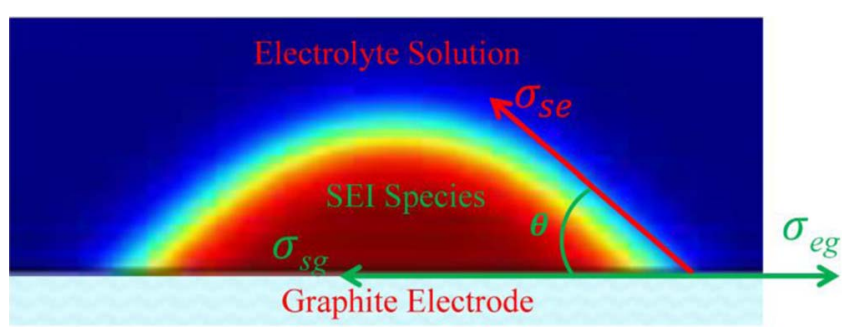

Figure 2. Schematic of contact angle with the surface tension coefficients of SEI species formed on anode surface. 


$$
\phi=-\nabla \cdot \varepsilon^{2} \nabla \varphi+\left(\varphi^{2}-1\right) \varphi
$$

Since the phase-field model does not require tracking of the interface, the chemical potential can be rewritten as a function of $\phi$, which is shown in Equation 12.

$$
\omega=\frac{\lambda}{\varepsilon^{2}} \phi
$$

Solid species deposit onto the electrode surface during formation and growth in the interfacial region between electrode and electrolyte. However, the phase-field model does not have the ability to describe the velocity of motion of the deposition process. Therefore, the NavierStokes equation is applied to capture the momentum balance: ${ }^{50,51,55}$

$$
\rho\left(\frac{\partial \mathbf{v}}{\partial t}+(\mathbf{v} \cdot \nabla) \mathbf{v}\right)=-\nabla P+\nabla \cdot\left[\mu\left(\nabla \mathbf{v}+(\nabla \mathbf{v})^{T}\right)\right]+F_{s t}
$$

where $\rho$ is the density of the electrolyte, $P$ is pressure in the electrolyte, $\mu$ is the dynamic viscosity of the electrolyte, and $F_{s t}$ is the surface tension force, as defined as below:

$$
F_{s t}=\frac{\lambda}{\varepsilon^{2}} \phi \cdot \nabla \varphi=\omega \nabla \varphi
$$

The boundary conditions of the electrolyte solution and SEI are shown below:

$$
\begin{gathered}
\mathbf{v}=\mathbf{v}_{0}=0 \quad P=P_{0}=0 \\
\mathbf{n} \frac{\gamma \lambda}{\varepsilon^{2}} \nabla \phi=0
\end{gathered}
$$$$
\mathbf{n} \varepsilon^{2} \nabla \varphi=\varepsilon^{2} \cos (\theta)|\nabla \varphi|
$$

where $\mathbf{n}$ is the unit vector normal to the boundary. The boundary condition of the outlet for the electrolyte solution is shown below,

$$
\left\{\mu\left(\nabla \mathbf{v}+(\nabla \mathbf{v})^{T}\right)\right\} \cdot \mathbf{n}=0
$$

Fick's law and mass balance are coupled with the phase-field simulation to investigate lithium-ion diffusion in different SEI layers: ${ }^{.6}$

$$
\frac{\partial C_{L i}(y)}{\partial t}+\nabla \cdot\left[-D_{i} \nabla C_{L i}(y)\right]=0
$$

Here, $C_{L i}(y)$ is the concentration of lithium ions at different locations $(y)$ in the SEI layer, and $D_{i}$ is the lithium-ion diffusion coefficient in SEI species $i$.

Then, the lithium-ion diffusion coefficient of SEI layer can be predicted by applying Fick's second law:

$$
\frac{\partial C_{i}}{\partial t}=\frac{\partial}{\partial y}\left(D \frac{\partial C_{i}}{\partial y}\right)
$$

where $D$ is the lithium-ion diffusion coefficient in a SEI layer consisting of various SEI species. The lithium-ion diffusion coefficient in the SEI layer can be represented as:

$$
D=\frac{1}{2 t}\left(\frac{d y}{d C_{i}}\right)_{y=\varepsilon} \int_{0}^{\varepsilon} y \frac{d C_{i}}{d y} d y
$$

In order to reduce the computational complexity of simulating numerous SEI species, X-ray photoelectron spectroscopy (XPS) experiments were carried out to identify the main components of SEI layer formed on a graphite surface. A graphite (MTI) composite anode (95:5 graphite/ PVDF) was used to fabricate Swagelok graphite/Li half cells that were cycled at $25^{\circ} \mathrm{C}$ and $50^{\circ} \mathrm{C}$. All cells were subjected to formation cycles that stopped at $1.0 \mathrm{~V}$. The cycled cells were disassembled and rinsed with dimethyl carbonate (DMC) for 15 seconds. The test electrodes were then sealed in a vessel under an argon atmosphere to transfer into the XPS sample chamber without exposure to air. The chemical composition of the SEI was investigated by XPS using a monochromatic Al $\mathrm{K}$-alpha $\mathrm{X}$-ray source with a scan area

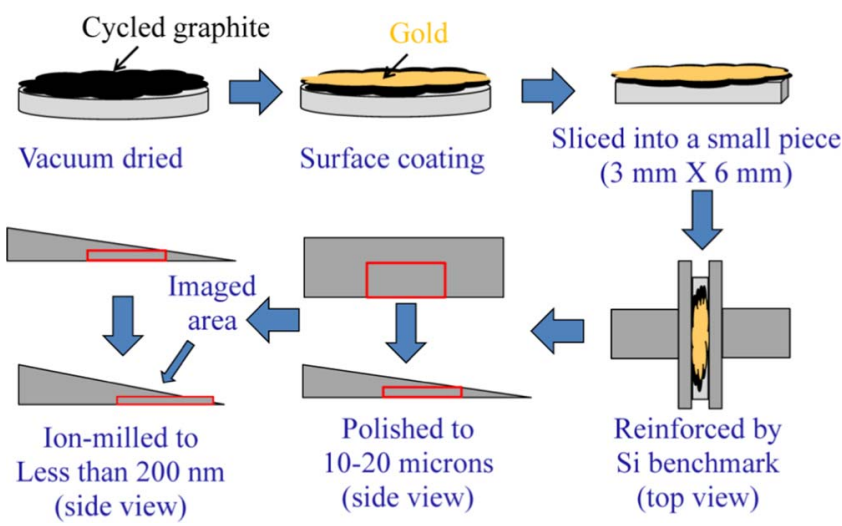

Figure 3. TEM sample preparation procedure.

of $700 \times 300 \mu \mathrm{m}$ of cycled graphite electrode. The resulting spectra were obtained by using repeated scans (more than 10 times) with a pass-energy of $20 \mathrm{eV}$ (high resolution) and $160 \mathrm{eV}$ (survey).

In addition, transmission electron microscopy (TEM) experimentation can be used to reveal the morphology of interfacial layers. However, TEM sample preparation is a delicate and time-consuming process. Firstly, a delicate procedure to prepare cross-sectional samples for TEM imaging with minimal damage during sample preparation needs to be developed. Figure 3 shows the procedure of TEM sample preparation. When preparing the TEM specimen, low angle and low Ar ion beam energy were used plus all the ion milling was performed at $\mathrm{LN}_{2}$ temperature. When TEM observation was performed, we minimized the beam current by spreading the beam and also the areas from which the images were taken were only exposed to the electron beam with very short time. All those operation should have reduced the damage to a very low level if not total reduced.

\section{Results and Discussion}

The phase-field model developed in the previous section is applied to capture SEI formation and morphology evolution. Detailed chemical constituents of SEI are not considered in this work. Instead, focus is placed on the evolution of SEI microstructure that is related to the electrochemical properties of interest (e.g., porosity and diffusivity).

The detailed description of SEI layer formation can be referred to Yan et al. ${ }^{31} \mathrm{X}$-ray photoelectron spectroscopy (XPS) experiments were performed to confirm major SEI species to be $\mathrm{LiF}, \mathrm{Li}_{2} \mathrm{O}, \mathrm{ROLi}$, and $\mathrm{ROCO}_{2} \mathrm{Li}$, as shown in Figure 4. The XPS analyses above show high-resolution XPS spectra of the SEI layer formed on a graphite surface. In F 1s spectrum, two peaks can be assigned. The peak near $686 \mathrm{eV}$, assigned to $\mathrm{LiF}$, is detected as a portion of SEI layer. LiF is also found in $\mathrm{Li} 1 \mathrm{~s}$ at approximately $56.4 \mathrm{eV}$. The residual $\mathrm{LiPF}_{6}$ or its reduction products $\mathrm{Li}_{\mathrm{x}} \mathrm{PF}_{\mathrm{y}}$ is formed on the graphite surface in $\mathrm{F} 1 \mathrm{~s}$ at approximately $688.1 \mathrm{eV}$. The small peaks near $532.8 \mathrm{eV}$ in $\mathrm{O} 1 \mathrm{~s}$ and $289.8 \mathrm{eV}$ in $\mathrm{C} 1 \mathrm{~s}$ correspond to the formation of inorganic $\mathrm{Li}_{2} \mathrm{CO}_{3}$ that can be easily decomposed into $\mathrm{Li}_{2} \mathrm{O}$. In addition, one of the main components of the SEI layer, the lithium alkyl carbonate $\mathrm{ROCO}_{2} \mathrm{Li}$, is formed due to the reduction of solvents. It can be obtained near $534.4 \mathrm{eV}$ in $\mathrm{O} 1 \mathrm{~s}$ and $287.9 \mathrm{eV}$ in $\mathrm{C} 1 \mathrm{~s}$.

Analysis of the TEM experiment results allow the porosity of the SEI to be estimated, as shown in Figure 5. The porosity of SEI close to the graphite surface is much smaller than that closer to the electrolyte solution, which indicates SEI may become more porous as it grows into the electrolyte. Figure 5 shows the SEI layer after cycling. The TEM image confirms that the SEI layer has a multilayered structure. The multilayered structure indicates either composition variation or thickness variation of the interphase layers.

Figure 6 shows the variation of chemical potential $(\omega)$ during SEI species formation above the graphite surface. Initially, the chemical potential exhibits no specific pattern. This is shown in Figure 6a 

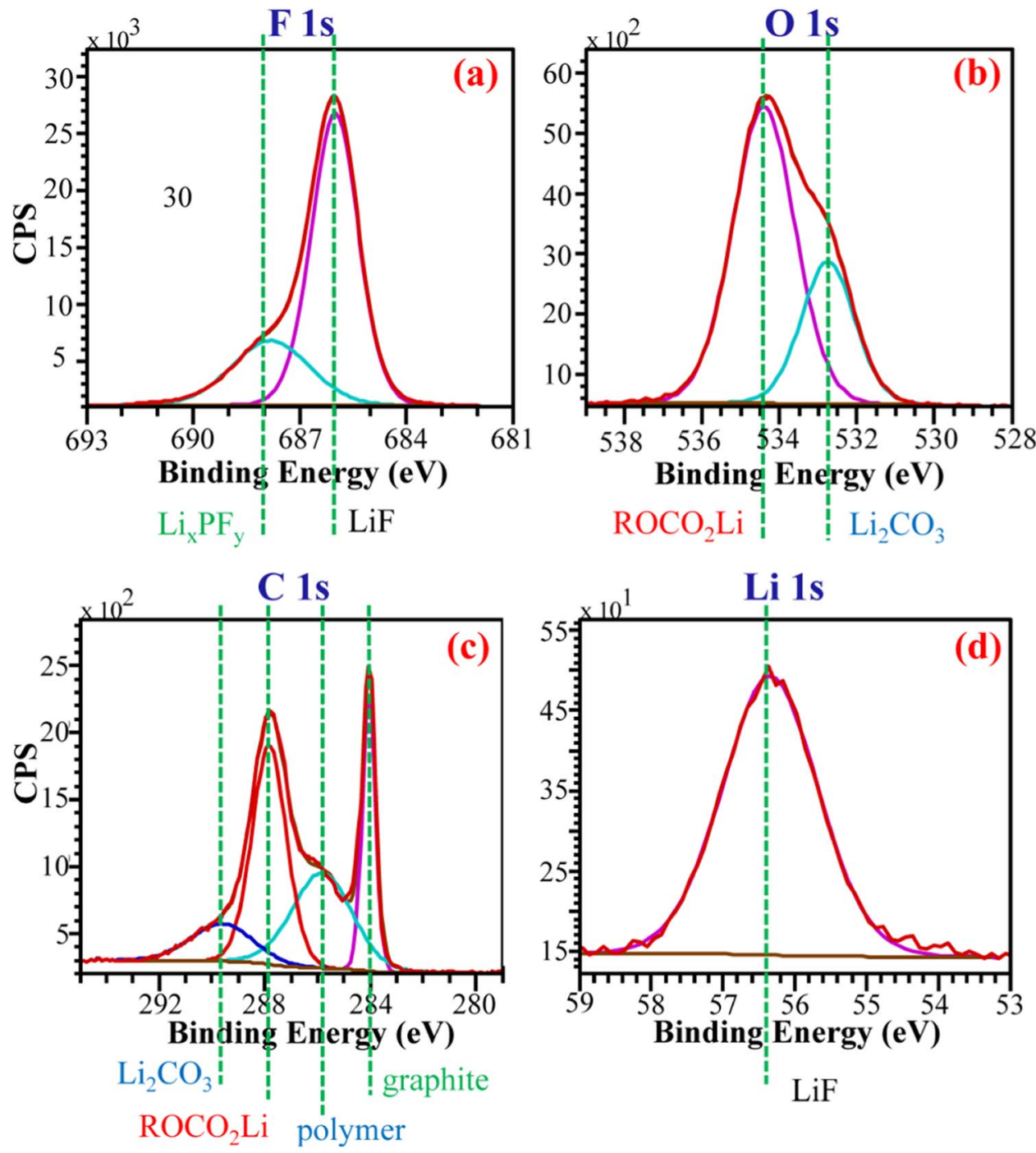

Figure 4. XPS spectra tests of cycled graphite to confirm $\mathrm{SEI}$ major species as $\mathrm{LiF}, \mathrm{Li}_{2} \mathrm{O}, \mathrm{ROLi}$, and $\mathrm{ROCO}_{2} \mathrm{Li}$.

where the SEI species have started to form and approach the graphite surface, but have not yet made contact. The double-well function is shown clearly in Figure $6 \mathrm{c}$ and $6 \mathrm{~d}$ with the spinodal region and twophase region during the formation process. ${ }^{47}$ Spinodals correspond to the curvature of the chemical potential is 0 when the diffusive interface reaches the equilibrium state. A uniform solid with a com- position between the spinodals is unstable and decomposes into a mixture of two phases. The phase-field variable continuously changes with the contact angle to minimize the total free energy density. Thus, according to Equation 9, the chemical potential from Figures $6 \mathrm{~b}, 6 \mathrm{c}$, and $6 \mathrm{~d}$ will also change during the SEI species morphology evolution.
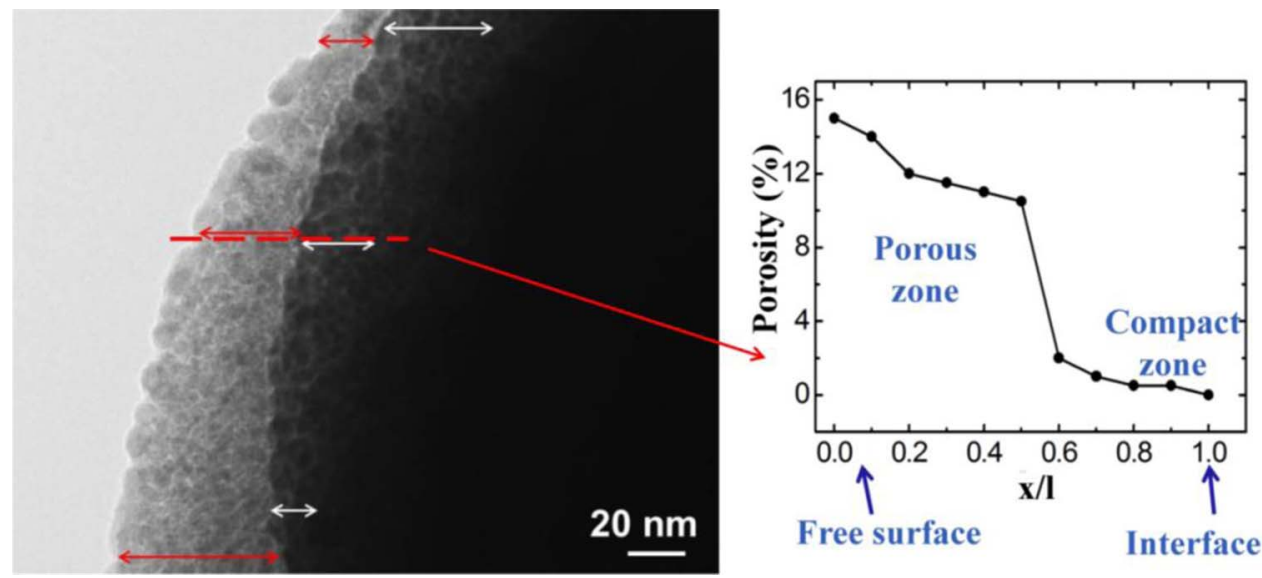

Free surface

Interface

Figure 5. TEM image and the porosity analysis of SEI layer. 

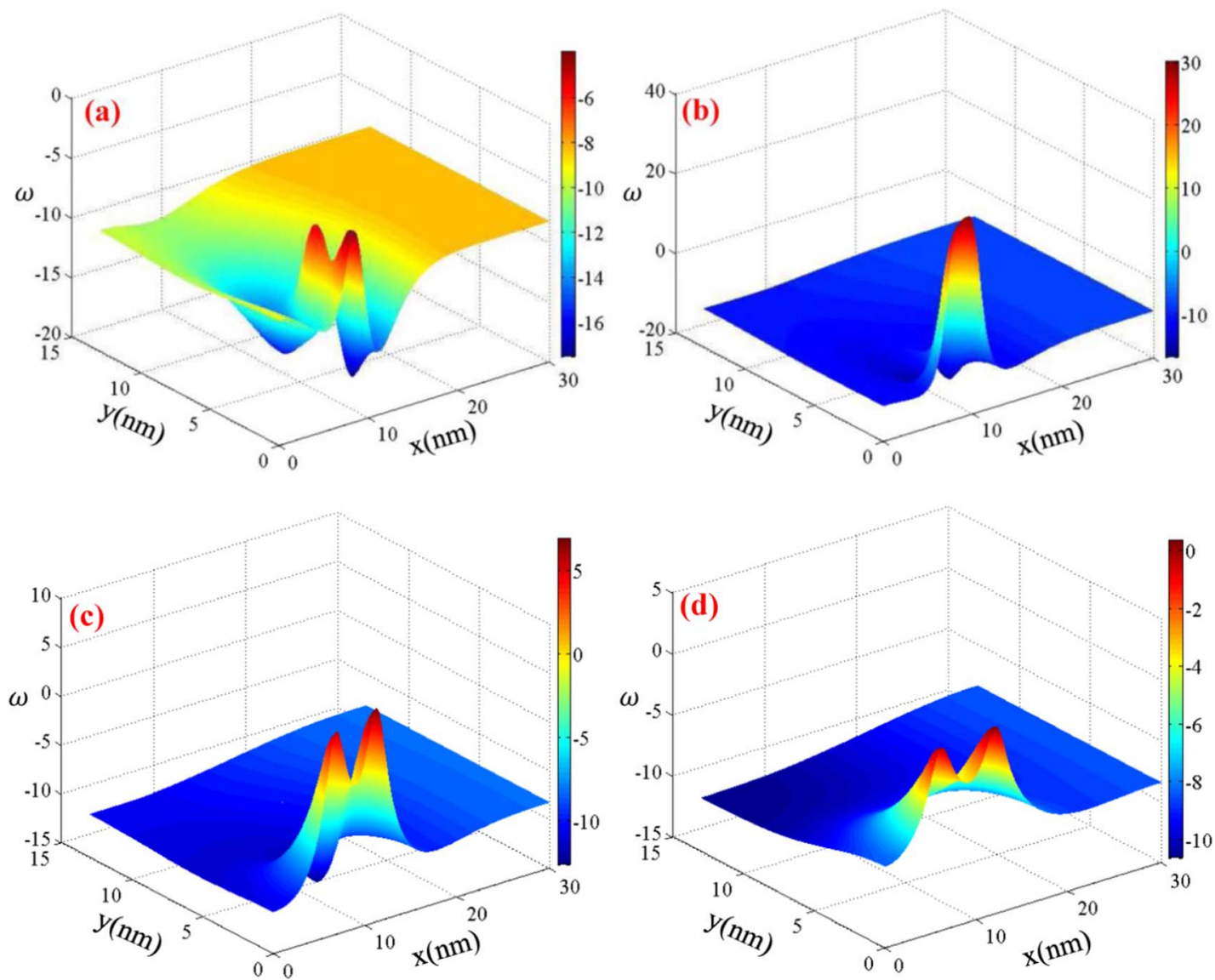

Figure 6. Variation of chemical potential $(\omega)$ in the electrolyte solution phase (a: $t=0.6 \mu \mathrm{s}, \mathrm{b}: \mathrm{t}=0.9 \mu \mathrm{s}, \mathrm{c}: \mathrm{t}=1.2 \mu \mathrm{s}, \mathrm{d}: \mathrm{t}=1.8 \mu \mathrm{s})$.

Figure 7 shows the evolution process of a single SEI species during the formation. The contact angle will determine the morphology evolution of a SEI species since it is assumed that the SEI/electrolyte interface always tends to keep a spherical configuration. Different materials make different contact angles since they have different surface and volume free energy. Additionally, the surface free energy density varies with time during the minimization. Thus, changes of SEI morphology are related to the variation of the total free energy density of SEI species. The total free energy density equation, Equation 2, governs the SEI species during the formation. Equations 8 and 9 are applied to control the minimization of the total free energy density. The surface free energy density is governed by the contact angles in Equation 6. This study is limited by the lack of experimental contact angles and total free energy density simulations for each SEI component above the graphite surface. Thus, all contact angles used are assumed values. Molecular dynamics (MD) simulations are ongoing to calculate contact angles of SEI species during SEI formation and growth.
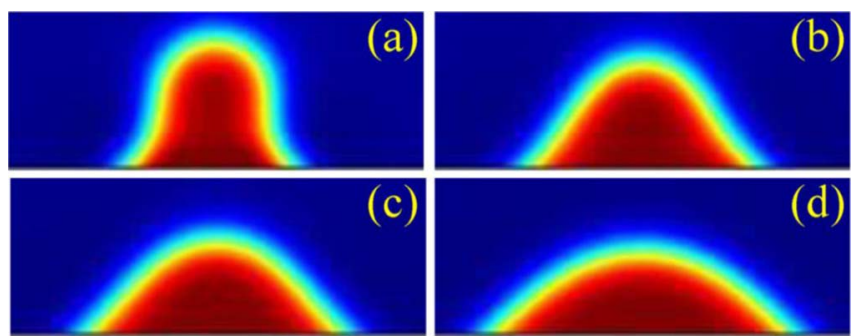

Figure 7. A single SEI species formation evolution at different times (a: $\mathrm{t}=$ $1.0 \mu \mathrm{s}, \mathrm{b}: \mathrm{t}=1.2 \mu \mathrm{s}, \mathrm{c}: \mathrm{t}=1.6 \mu \mathrm{s}, \mathrm{d}: \mathrm{t}=1.8 \mu \mathrm{s})$.
Figure 8 further extends morphology evolution of SEI species in two dimensions by showing the variation of the phase-field variable during SEI species formation and evolution above the graphite surface. As indicated by the color change in Figure 8, the phase-field variable varies smoothly from 1 to -1 . Recall that the phase-field variable is representative of solid species (SEI) and liquid species (electrolyte solution) when $\varphi=1$ and -1 , respectively. ${ }^{41,42}$ Figure 8 also connects the contact angle and phase-field variable together to show changes in the interface region between the solid SEI species and electrolyte solution. As mentioned previously, different surface free energy densities will form different contact angles. Figures 6 and 8 clearly show the effect of chemical potential and surface free energy density on SEI formation and morphology evolution.

Due to the repetition of the formation process shows in Figure 7, a SEI layer growth and morphology evolution simulation results are shown in Figure 9. The Navier-Stokes equation is applied to capture the momentum balance during the growth. Different contact angles are assumed between different SEI species, as well as between SEI species and the graphite surface. Based on the simulated SEI morphology evolution, the thickness growth rate of SEI layer can be estimated, as shown in Figure 10. From Figure 10, it can be seen that the SEI layer grows quickly at first. This occurs because the concentration of charged species at the interface is initially high. As the passivation layer grows, it hinders direct contact between the electrolyte solution and the graphite electrode. Since the porous SEI layer is conductive to lithium ions but nonconductive to electrons, the resultant decomposed species become unable to react with electrons from the electrode to be further reduced. As a result, the SEI growth rate will decrease due to the low concentration of electrons at the interface.

Simulation of different types of SEI microstructures are shown in the figures below. Figure 11a and Figure 11b show the structure of the compact and porous SEI layer, respectively. Figure 11c shows the 

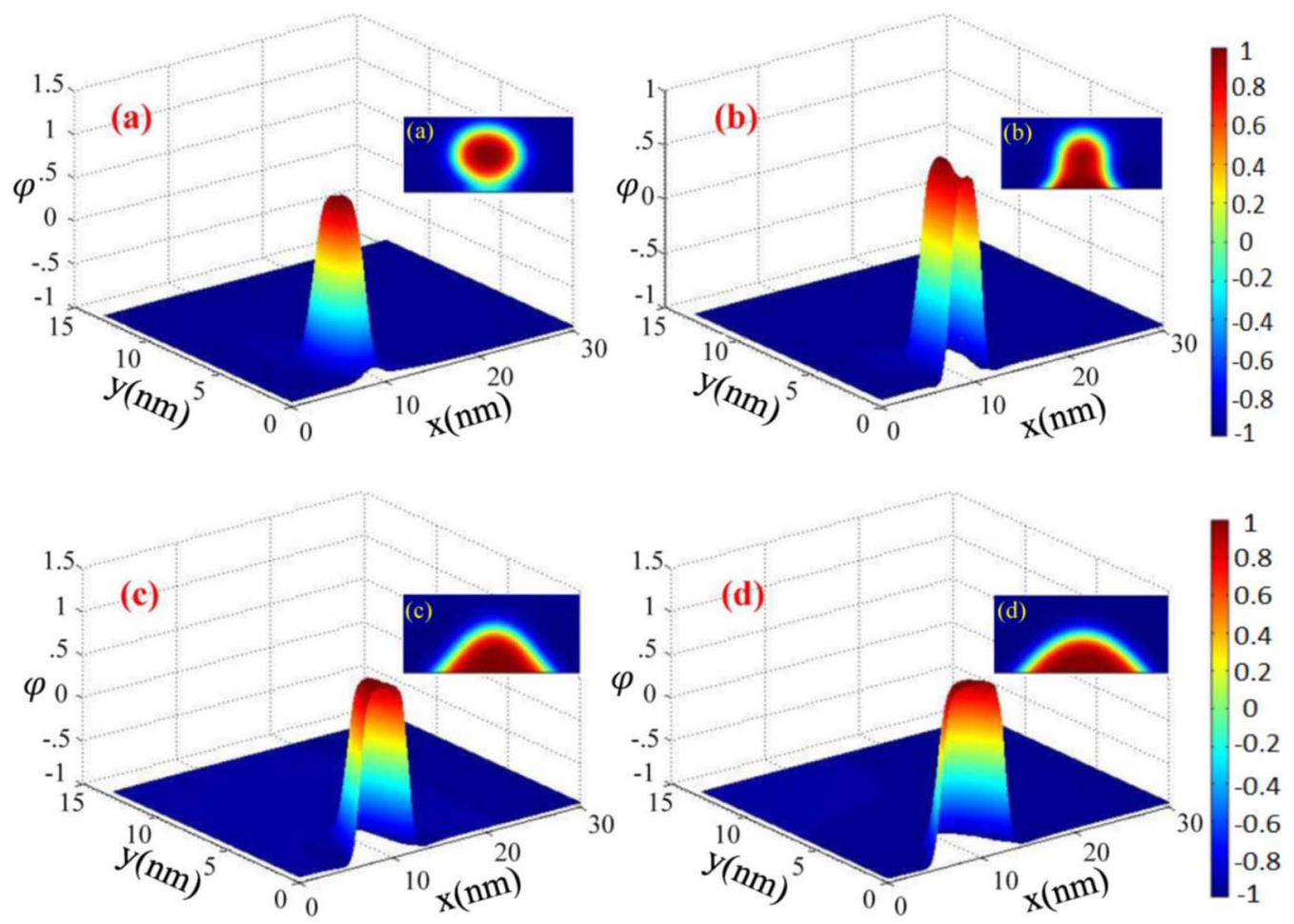

Figure 8. Variation of phase-field variable $(\varphi)$ in the electrolyte solution during the formation of a single SEI species $(\mathrm{a}: \mathrm{t}=0.6 \mu \mathrm{s}, \mathrm{b}: \mathrm{t}=0.9 \mu \mathrm{s}, \mathrm{c}: \mathrm{t}=1.2 \mu \mathrm{s}$, $\mathrm{d}: \mathrm{t}=1.8 \mu \mathrm{s})$.

multilayered structure of SEI. In order to simplify the complexity of simulation, SEI species in the compact and porous layers are assumed to be pure inorganic and organic materials, respectively. Therefore, each layer has a different material composition. Recall that different materials are characterized by specific surface and volume free energy densities. Since the surface free energy density determines the contact angle, using different assumed contact angles will simulate different SEI layer morphologies. First, $\mathrm{LiPF}_{6}$ in the electrolyte solution decomposes to produce solid species, such as $\mathrm{LiF},\left(\mathrm{CH}_{2} \mathrm{CH}_{2} \mathrm{O}\right)_{2}$, and $\left(\mathrm{CH}_{2} \mathrm{OCOOCH}_{3}\right)_{2}$. However, no solid particles form on the graphite surface initially. As the concentration of decomposition products increases, the resultant solid species precipitate from the electrolyte solution. These species are substituted by $\mathrm{LiF}$, and $\mathrm{Li}_{2} \mathrm{CO}_{3}$. In addition, $\mathrm{Li}_{2} \mathrm{CO}_{3}$ species may easily be substituted by $\mathrm{LiF}$ and $\mathrm{Li}_{2} \mathrm{O}$. Eventually, the components of the films near to the graphite electrode surface are entirely $\mathrm{LiF}$ and $\mathrm{Li}_{2} \mathrm{O} .^{31}$ Meanwhile, the components of the films far away from the electrode surface are organic materials: $\mathrm{ROLi}$ and $\mathrm{ROCO}_{2} \mathrm{Li}$.

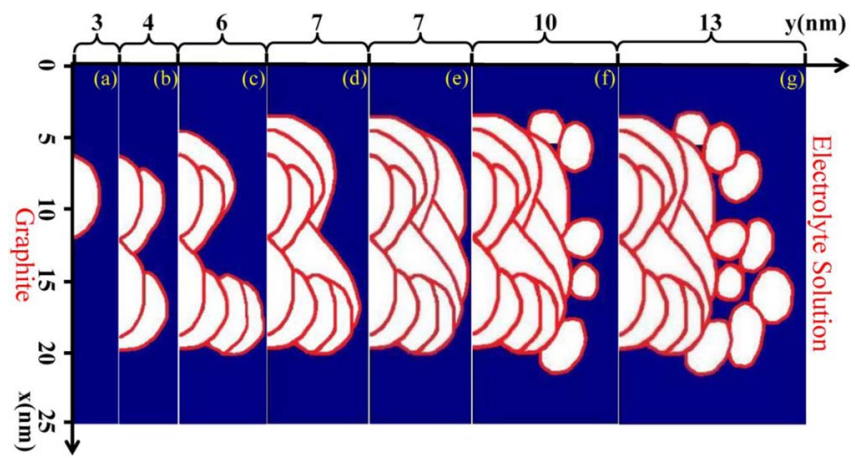

Figure 9. Morphology evolution during SEI growth $(\mathrm{a}: \mathrm{t}=1.8 \mu \mathrm{s}, \mathrm{b}: \mathrm{t}=7.2$ $\mu \mathrm{s}, \mathrm{c}: \mathrm{t}=12.6 \mu \mathrm{s}, \mathrm{d}: \mathrm{t}=16.2 \mu \mathrm{s}, \mathrm{e}: \mathrm{t}=19.8 \mu \mathrm{s}, \mathrm{f}: \mathrm{t}=28.8 \mu \mathrm{s}, \mathrm{g}: \mathrm{t}=36 \mu \mathrm{s})$.
In addition, the developed model is capable of tracing every SEI species to determine its location during SEI layer growth, as shown in Figure 12 . This ability makes it possible to investigate the properties of the SEI layers.

The diffusion model is applied to the SEI layer simulated by phasefield model in order to predict lithium-ion concentration distribution. $D_{i}$ is the lithium-ion diffusion coefficient in different SEI species $i$, as shown in Table I. In this work, three temperatures, $298 \mathrm{~K}$ (the reference temperature, $T_{r e f}$ ), $308 \mathrm{~K}$, and $318 \mathrm{~K}$, are used to investigate the lithium-ion concentration distributions and diffusion coefficients in different types of SEI layers. $D_{i}$ at $298 \mathrm{~K}$ is obtained from the work of Ken et al. ${ }^{57}$ The Arrhenius equation, below, is applied to determine

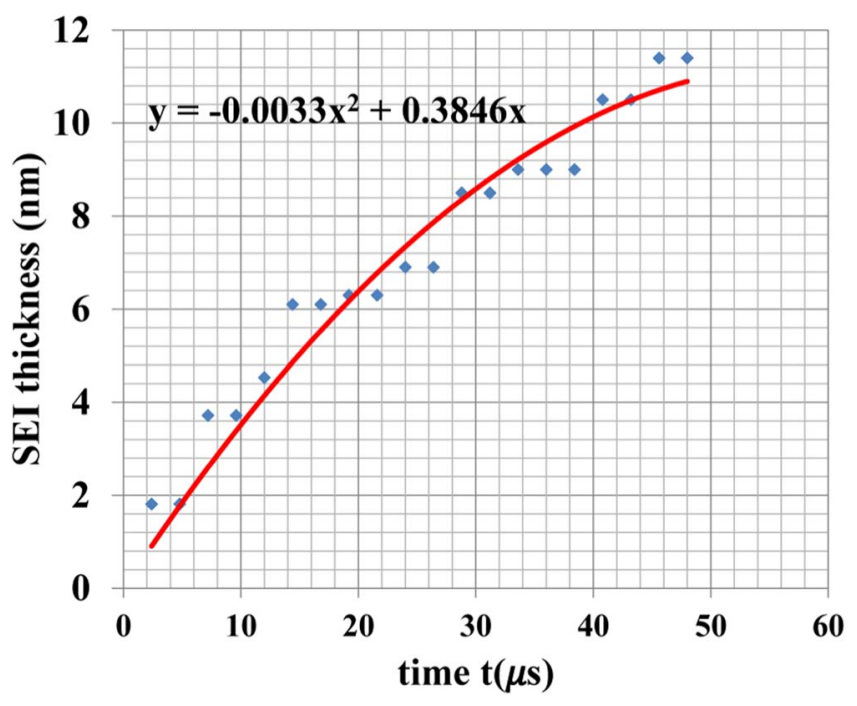

Figure 10. The thickness growth rate of SEI layer. 

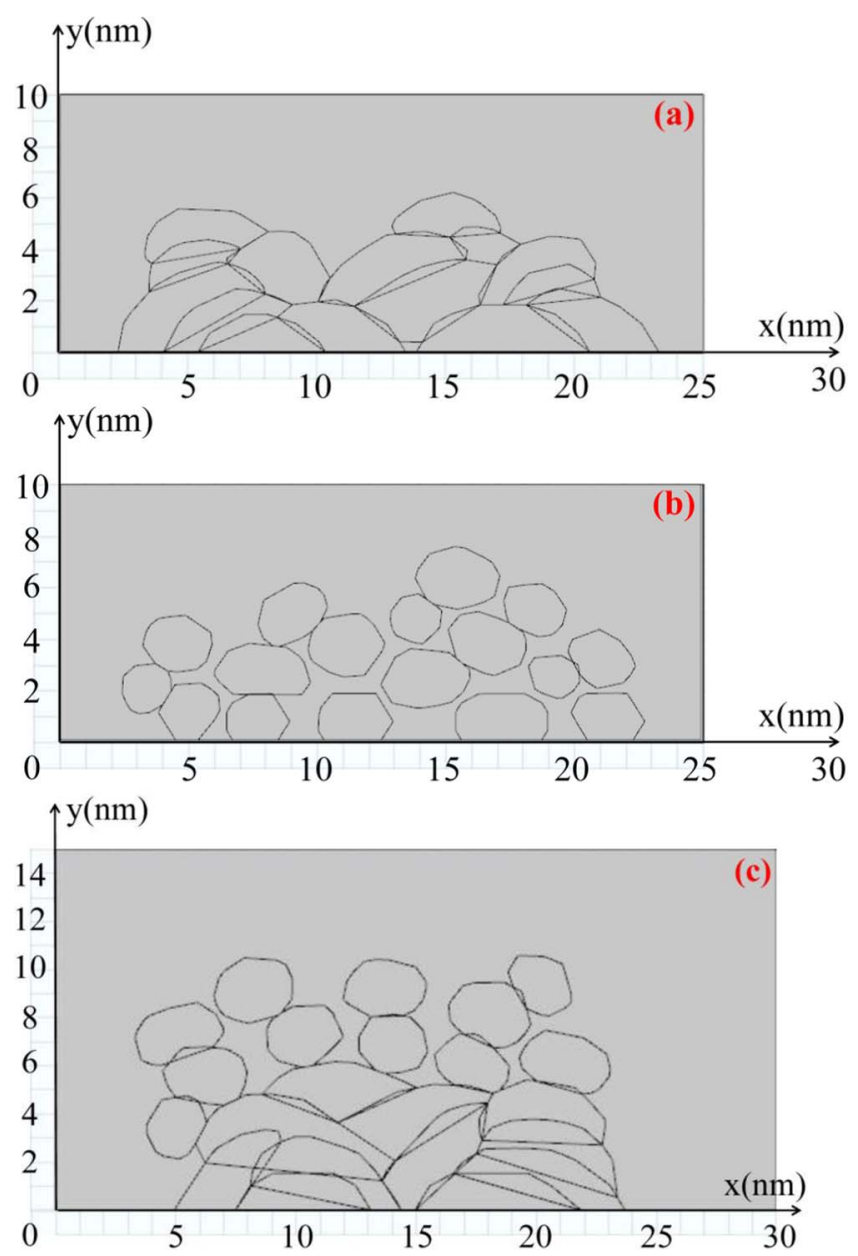

Figure 11. Simulation of (a) compact structure of SEI layer, (b) porous structure of SEI layer, and (c) multilayered structure of SEI layer.

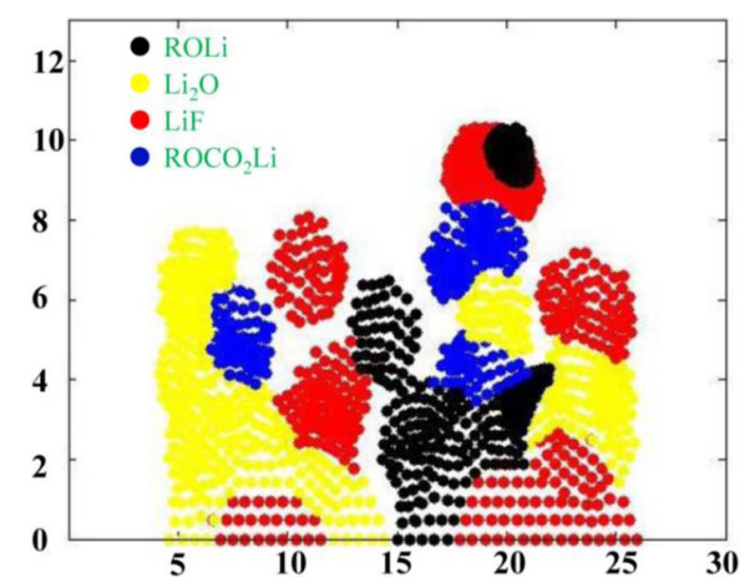

Figure 12. Simulation of SEI species location and distribution during SEI formation and growth.
$D_{i}$ at $308 \mathrm{~K}$ and $318 \mathrm{~K}$, as shown in Table $\mathrm{I}^{38}$

$$
D_{i}=D_{\text {ref }} \exp \left[\frac{E_{a c t, D_{i}}}{R}\left(\frac{1}{T_{\text {ref }}}-\frac{1}{T}\right)\right]
$$

Here $\mathrm{R}$ is the universal gas constant, and $D_{\text {ref }}$ is the reference lithiumion diffusion coefficient in each SEI species $i$ at reference temperature $T_{\text {ref. }} E_{\text {act }}$ is the corresponding activation energy, which is $4 \mathrm{~kJ} / \mathrm{mol}$ and $10 \mathrm{~kJ} / \mathrm{mol}$ for the solid and liquid phases, respectively. ${ }^{38}$

Figure 13 shows the lithium-ion concentration distributions in the compact, porous, and multilayered structure of SEI layer. The paths of lithium-ion diffusion in these three kinds of SEI layers are shown in Figure 14. The lithium ions are assumed to diffuse through the electrolyte solution into SEI layer normal to the graphite surface. After a certain time, the differences in lithium-ion concentration between the electrolyte and graphite surface for SEI compact and porous layers are around $550 \mathrm{~mol} / \mathrm{m}^{3}$ and $163 \mathrm{~mol} / \mathrm{m}^{3}$, respectively, as shown in Figure $13 \mathrm{a}$ and $13 \mathrm{~b}$. In addition, this difference reaches nearly 795 $\mathrm{mol} / \mathrm{m}^{3}$ for multilayered structure of SEI. This indicates that SEI layer has a significant effect on the lithium-ion diffusion during the first electrochemical intercalation. SEI layer essentially slows down the lithium-ion diffusion from the electrolyte solution into the graphite. Lithium ions need more time to pass through the SEI compact layer than the porous layer, which indicates that the lithium-ion diffusion coefficient in the compact SEI layer is smaller than the porous layer. The blue lines in Figure 14 outline the paths of lithium-ion diffusion. These does not change much in the porous SEI layer. This is due to the fact that the diffusion coefficients of the organic species are very similar to the electrolyte solution and the porous sites contain electrolyte solution.

As lithium ions pass through the SEI layer and reach the graphite surface, the lithium-ion diffusion coefficient in the SEI layer can be calculated using Fick's second law. The detailed calculation can be referred to our previous work. ${ }^{52}$ Calculated diffusion coefficients at various temperatures are shown in Figure 15. The lithium-ion diffusion coefficients increase with rising temperatures in all three SEI structures. In addition, different SEI structures influence lithium-ion diffusion and result in different diffusion coefficients. The order of magnitude of diffusion coefficients are $10^{-16} \mathrm{~cm}^{2} / \mathrm{s}, 10^{-12} \mathrm{~cm}^{2} / \mathrm{s}$, and $10^{-15} \mathrm{~cm}^{2} / \mathrm{S}$ in the compact, porous and multilayered structures of SEI layer, respectively. This indicates that lithium ions can pass through the porous layer more easily than the compact layer. To date, there are very few studies on experimental measurement of diffusion coefficients in compact, porous, and multilayered structures of SEI due to the complexity of both controlling SEI layer formation and growth and experimentation in SEI layer. However, there are some computational studies on diffusion coefficients published recently. ${ }^{58-61}$ Pinson and Bazant ${ }^{59}$ calculated diffusion coefficient for homogenous SEI layer. In their model, the diffusion coefficient $\mathrm{D}$ is $3\left(10^{-16}\right) \mathrm{cm}^{2} / \mathrm{s}$ at $60^{\circ} \mathrm{C}$. They also indicated that the true value of $\mathrm{D}$ is higher than that calculated valued due to the assumptions such as treating SEI layer as homogenous structure. Their final estimated value of $D$ is $4\left(10^{-15}\right) \mathrm{cm}^{2} / \mathrm{s}$. We adopted the input from Pinson and Bazant and found the diffusion coefficient is around $2.583\left(10^{-15}\right) \mathrm{cm}^{2} / \mathrm{s}$ at $60^{\circ} \mathrm{C}$ that is in same order of magnitude with Pinson and Bazant. ${ }^{59}$ With incorporating more SEI growth mechanism and related experimental studies, we can address this model validation and comparison more appropriately. Otherwise, it is difficult to compare computational results with different models due to the different assumptions adopted. All of these assumptions can significantly affect the calculation of diffusion coefficients.

Table I. The lithium-ion diffusion coefficients $\left(\mathrm{cm}^{2} / \mathrm{s}\right)$ in different materials at different temperatures (K).

\begin{tabular}{cccccc} 
& $\mathrm{Li}_{2} \mathrm{O}$ & $\mathrm{LiF}$ & $\mathrm{ROLi}$ & $\mathrm{ROCO}_{2} \mathrm{Li}$ & Electrolyte \\
\hline 298 & $1.6 \times 10^{-16}$ & $3.5 \times 10^{-16}$ & $1.1 \times 10^{-11}$ & $7 \times 10^{-12}$ & $8 \times 10^{-12}$ \\
308 & $1.686 \times 10^{-16}$ & $3.688 \times 10^{-16}$ & $1.159 \times 10^{-11}$ & $7.377 \times 10^{-12}$ & $9.120 \times 10^{-12}$ \\
318 & $1.771 \times 10^{-16}$ & $3.874 \times 10^{-16}$ & $1.218 \times 10^{-11}$ & $7.748 \times 10^{-12}$ & $1.031 \times 10^{-11}$
\end{tabular}




\section{Concentration Distribution $\mathrm{C}\left(\mathrm{mol} / \mathrm{m}^{3}\right)$}

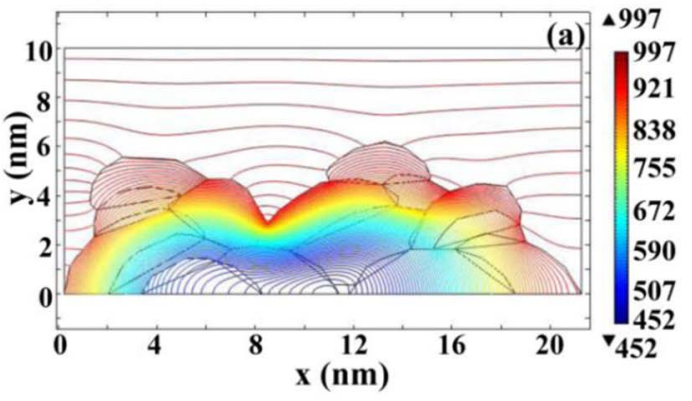

Concentration Distribution $\mathrm{C}\left(\mathrm{mol} / \mathrm{m}^{3}\right)$

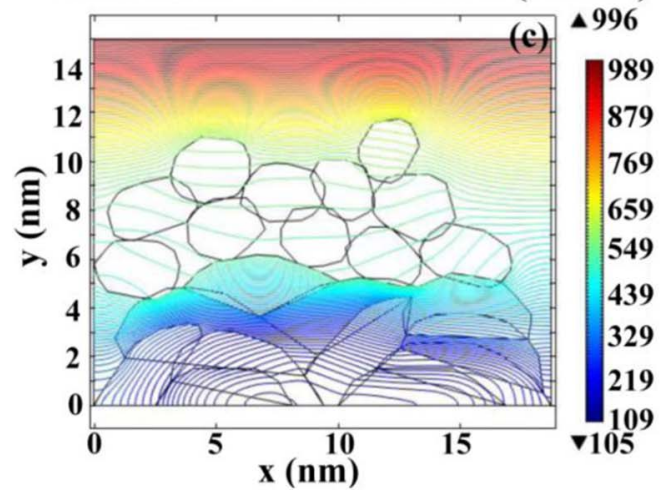

\section{Concentration Distribution $\mathrm{C}\left(\mathrm{mol} / \mathrm{m}^{3}\right)$}

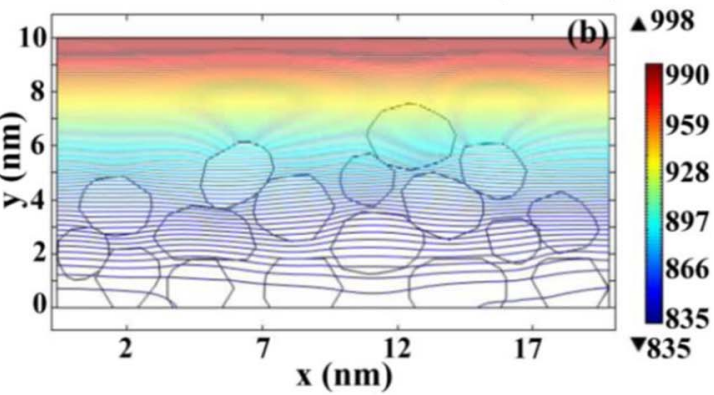

Figure 13. Lithium-ion concentrations in (a) compact, (b) porous, and (c) multilayered structure of SEI layer.

Moreover, SEI layer growth can cause cell internal resistant increase. Here, we assume the resistance of SEI layer is proportional to its thickness. ${ }^{36,38,59}$ Adopting our previous studies, ${ }^{36,38}$ the resistance of SEI layer can be estimated by $R_{S E I}=\sum_{i=1}^{N} \delta / \sigma_{i}$, where $\delta$ is
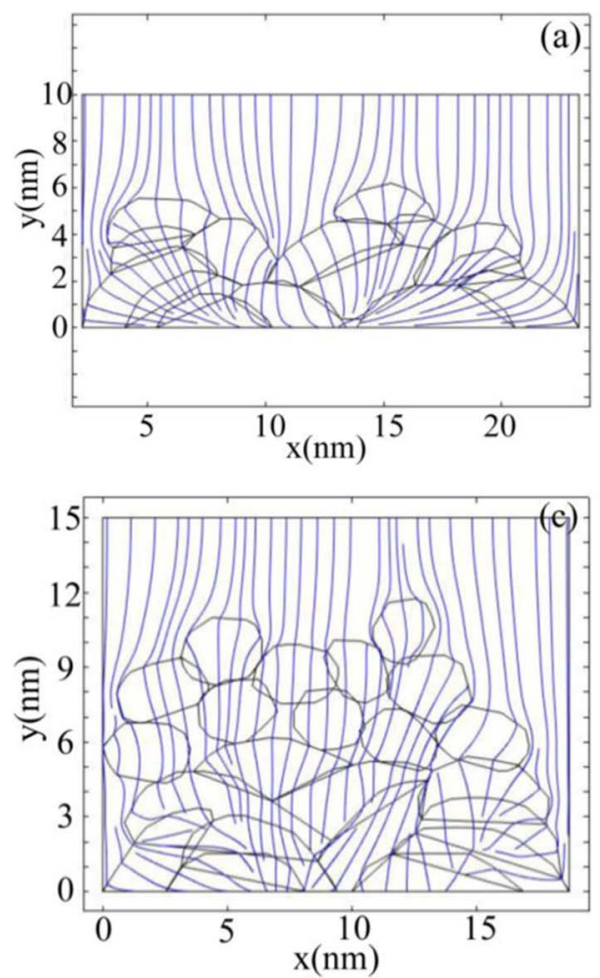

the thickness of SEI layer, and $\sigma_{i}$ is the ionic conductivity of each SEI species $i$. $\mathrm{N}$ is 4 in our work since SEI is simplified with four main components based on the XPS experiment. $\sigma_{i}$ at $298 \mathrm{~K}$ is estimated from the work of S. J. Harris et al. ${ }^{57}$ The Arrhenius equation $\sigma_{i}=\sigma_{r e f} \exp \left[\frac{E_{a c t}}{R}\left(\frac{1}{T_{r e f}}-\frac{1}{T}\right)\right]$ is applied to determine $\sigma_{i}$ at $308 \mathrm{~K}$

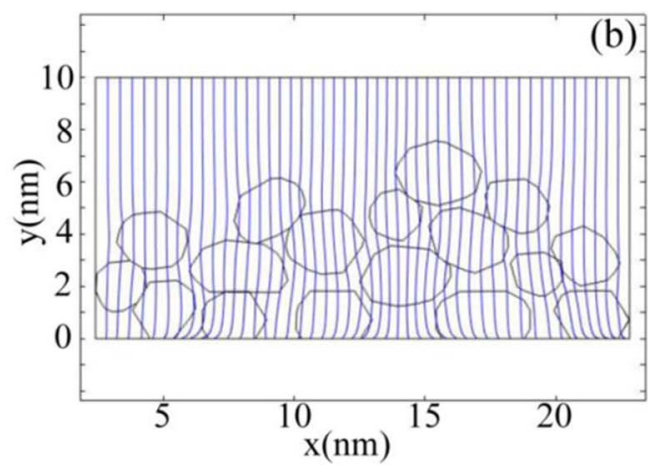

Figure 14. Lithium-ion diffusion paths in (a) compact, (b) porous, and (c) multilayered structure of SEI layer. 

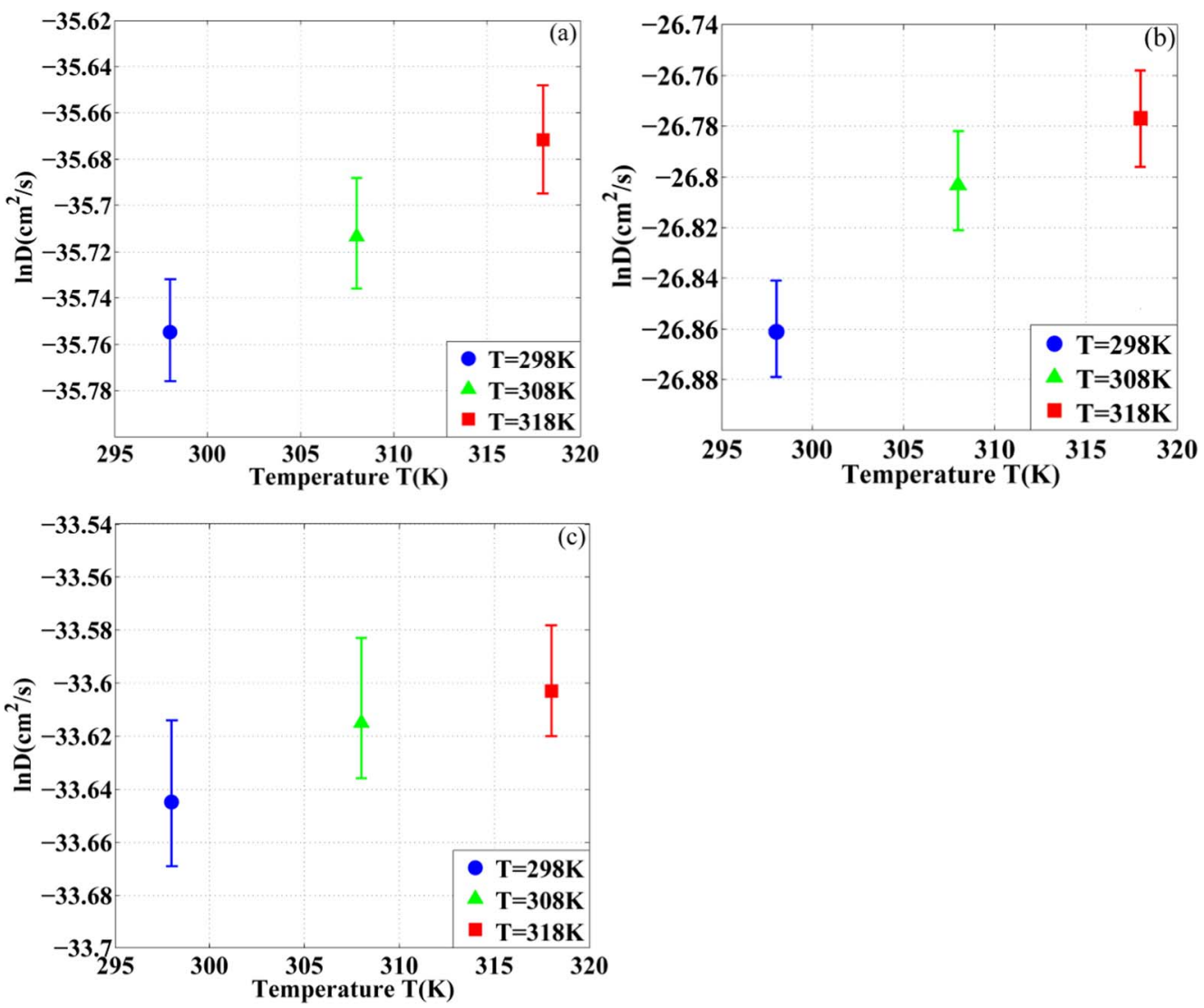

Figure 15. Lithium-ion diffusion coefficients in (a) compact, (b) porous, and (c) multilayered structure of SEI layer at different temperatures.
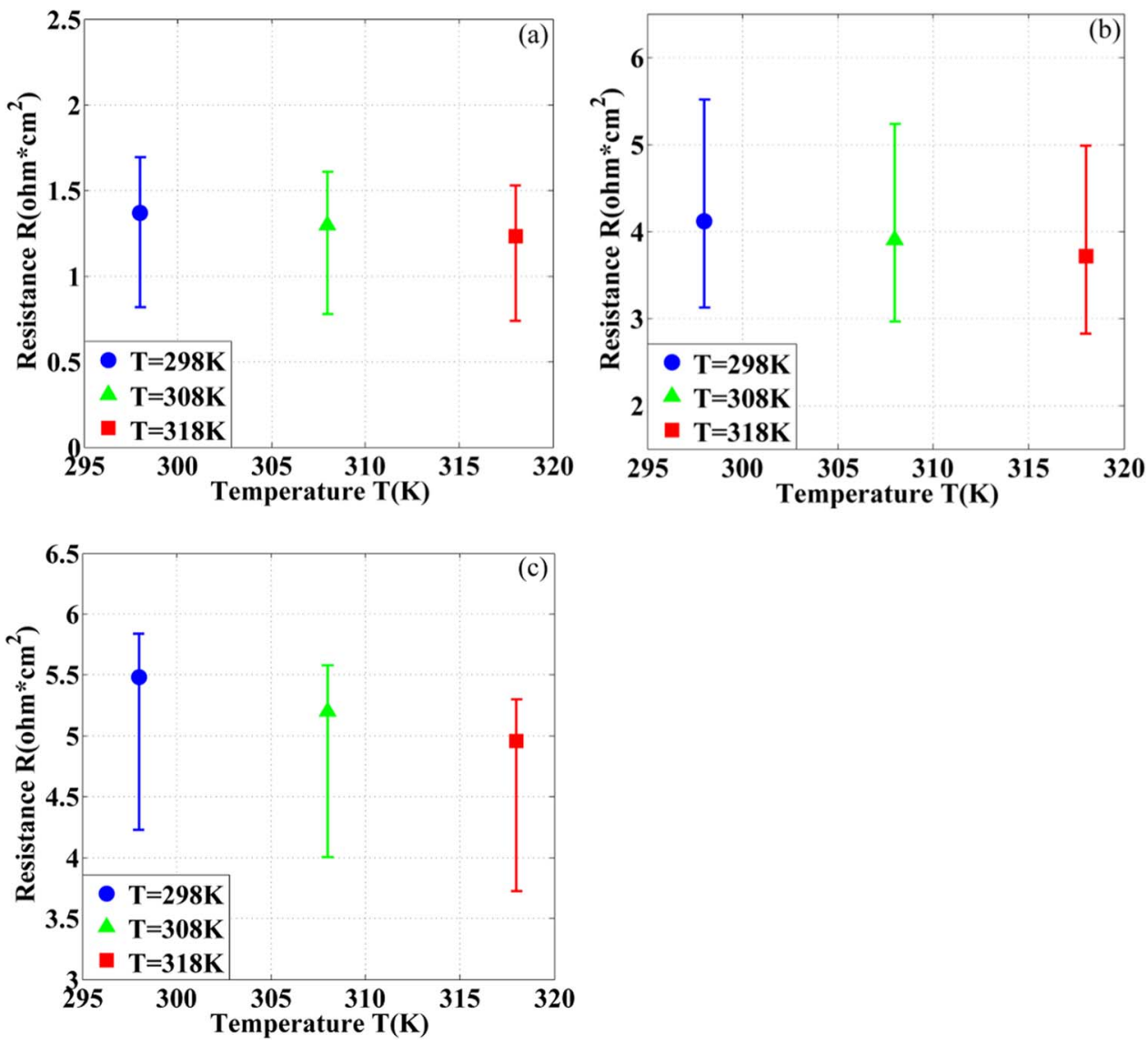

Figure 16. Resistances in (a) compact, (b) porous, and (c) multilayered structure of SEI layer at different temperatures. 
Table II. The ionic conductivity $(\mathrm{S} / \mathrm{cm})$ of different SEI species at different temperatures $(\mathbf{K})$.

\begin{tabular}{ccccc}
$T$ & $\mathrm{Li}_{2} \mathrm{O}$ & $\mathrm{LiF}$ & $\mathrm{ROLi}$ & $\mathrm{ROCO}_{2} \mathrm{Li}$ \\
\hline 298 & $4.102 \times 10^{-7}$ & $3.221 \times 10^{-7}$ & $1.403 \times 10^{-7}$ & $1.026 \times 10^{-7}$ \\
308 & $4.323 \times 10^{-7}$ & $3.394 \times 10^{-7}$ & $1.479 \times 10^{-7}$ & $1.081 \times 10^{-7}$ \\
318 & $4.540 \times 10^{-7}$ & $3.565 \times 10^{-7}$ & $1.553 \times 10^{-7}$ & $1.136 \times 10^{-7}$
\end{tabular}

and $318 \mathrm{~K}$, as shown in Table II. Here $\sigma_{r e f}$ is the reference ionic conductivity of each SEI species at reference temperature $T_{r e f} . E_{a c t}$ is the corresponding activation energy, which is $4 \mathrm{~kJ} / \mathrm{mol}$ for solid phase. ${ }^{38}$ Calculated resistances at various temperatures are shown in Figure 16. The resistances decrease with rising temperatures for all three different SEI structures. The resistances between $298 \mathrm{~K}$ and $318 \mathrm{~K}$ are $0.740-1.693 \Omega \cdot \mathrm{cm}^{2}, 2.827-5.517 \Omega \cdot \mathrm{cm}^{2}$, and 3.726-5.839 $\Omega \cdot \mathrm{cm}^{2}$ in the compact, porous, and multilayered structures of SEI layer, respectively.

\section{Conclusions}

A phase-field model was developed to investigate the formation and growth of SEI layers on the surface of a graphite anode. In this model, the shape of the SEI species is determined by minimizing the total free energy density of the two-phase system to reach a state of equilibrium. The chemical potential $(\omega)$ is satisfied by the double-well function. Compared with other models, the phase-field model does not need to track the interfaces directly. It also reveals the profiles of chemical potential and velocity in the electrolyte/SEI interfacial region. An important advantage of the present model compared with previous models is that it predicts the SEI layer structures in two-dimensions. Additionally, the developed model can capture the SEI growth and morphology evolution as well. A diffusion model is then applied to investigate the lithium-ion diffusion in different SEI layers. Fick's law and mass balance are applied to investigate the lithium-ion concentration distributions and diffusion coefficients in the compact, porous and multilayered structures of SEI predicted by the phase-field model. The simulation results show that lithium-ion diffusion coefficients between $298 \mathrm{~K}$ and $318 \mathrm{~K}$ are in the range of $1.340-7.328\left(10^{-16}\right) \mathrm{cm}^{2} / \mathrm{s}$, $1.734-3.405\left(10^{-12}\right) \mathrm{cm}^{2} / \mathrm{s}$, and $2.611-2.389\left(10^{-15}\right) \mathrm{cm}^{2} / \mathrm{s}$ in the compact, porous and multilayered structures of SEI layer, respectively. The resistances decrease with rising temperatures for all three different SEI structures. The resistances between $298 \mathrm{~K}$ and $318 \mathrm{~K}$ are $0.740-1.693 \Omega \cdot \mathrm{cm}^{2}, 2.827-5.517 \Omega \cdot \mathrm{cm}^{2}$, and $3.726-5.839 \Omega \cdot \mathrm{cm}^{2}$ in the compact, porous, and multilayered structures of SEI layer, respectively. Most importantly, the developed model has great potential to be extended to three dimensional space for SEI layer growth investigations. In addition, the developed phase-field model may be applied to other interphase growth and morphology evolution studies. Additional species and mechanisms relevant to SEI growth could be coupled with and analyzed by this model. The developed model could also be used to study interphase problems with complex morphology evolutions. Further development of the presented model will strengthen understanding of SEI microstructure evolution.

\section{Acknowledgments}

The present efforts were supported by the University of Kansas General Research Fund. The authors appreciate the help with experiments from Dr. Kai Sun (Department of Material Science \& Engineering) and Hosop Shin (Department of Mechanical Engineering) of the University of Michigan.

\section{References}

1. P. Arora, R. E. White, and M. Doyle, Journal of the Electrochemical Society, 145, 3647 (1998)

2. A. Barré, B. Deguilhem, S. Grolleau, M. Gérard, F. Suard, and D. Riu, Journal of Power Sources, 241, 680 (2013)
3. S. Paul, C. Diegelmann, H. Kabza, and W. Tillmetz, Journal of Power Sources, 239, 642 (2013).

4. E. Cho, J. Mun, O. B. Chae, O. M. Kwon, H.-T. Kim, J. H. Ryu, Y. G. Kim, and S. M. Oh, Electrochemistry Communications, 22, 1 (2012).

5. A. Abouimrane, J. Ding, and I. J. Davidson, Journal of Power Sources, 189, 693 (2009).

6. S. S. Zhang and T. R. Jow, Journal of Power Sources, 109, 458 (2002)

7. M. Jana, A. Sil, and S. Ray, Journal of Physics and Chemistry of Solids, 75, 60 (2014).

8. M. M. Thackeray, J. T. Vaughey, C. S. Johnson, A. J. Kropf, R. Benedek, L. M. L. Fransson, and K. Edstrom, Journal of Power Sources, 113, 124 (2003).

9. A. V. Virkar, Journal of Power Sources, 196, 5970 (2011).

10. K. Maher and R. Yazami, Journal of Power Sources, 247, 527 (2014).

11. L. Terborg, S. Weber, F. Blaske, S. Passerini, M. Winter, U. Karst, and S. Nowak, Journal of Power Sources, 242, 832 (2013).

12. R. Younesi, M. Hahlin, M. Roberts, and K. Edström, Journal of Power Sources, 225 40 (2013).

13. B. Li, M. Xu, T. Li, W. Li, and S. Hu, Electrochemistry Communications, 17, 92 (2012).

14. X. J. Wang, H. S. Lee, H. Li, X. Q. Yang, and X. J. Huang, Electrochemistry Communications, 12, 386 (2010).

15. H. Buqa, A. Würsig, J. Vetter, M. E. Spahr, F. Krumeich, and P. Novák, Journal of Power Sources, 153, 385 (2006).

16. E. Peled, D. Bar Tow, A. Merson, A. Gladkich, L. Burstein, and D. Golodnitsky, Journal of Power Sources, 97-98, 52 (2001).

17. H. Jannesari, M. D. Emami, and C. Ziegler, Journal of Power Sources, 196, 9654 (2011).

18. X. Lin, J. Park, L. Liu, Y. Lee, A. M. Sastry, and W. Lu, Journal of The Electrochemical Society, 160, A1701 (2013).

19. G. X. Wang, V. Prasad, and E. F. Matthys, Materials Science and Engineering: A, 225, 47 (1997).

20. M. Safari, M. Morcrette, A. Teyssot, and C. Delacourt, Journal of the Electrochemical Society, 156, A145 (2009).

21. P. Verma and P. Novák, Carbon, 50, 2599 (2012).

22. F. M. Wang, M. H. Yu, Y. J. Hsiao, Y. Tsai, B. J. Hwang, Y. Y. Wang, and C. C. Wan, International Journal of Electrochemical Science, 6, 1014 (2011).

23. M. H.-M. Tang, University of California, Berkeley, 1 (2012).

24. A. M. Colclasure, K. A. Smith, and R. J. Kee, Electrochimica Acta, 58, 33 (2011).

25. R. Srinivasan and B. G. Carkhuff, Journal of Power Sources, 241, 560 (2013).

26. G. K. Prasad and C. D. Rahn, Journal of Power Sources, 232, 79 (2013).

27. O. Borodin, G. D. Smith, and P. Fan, J Phys Chem B, 110, 22773 (2006).

28. M. Broussely, S. Herreyre, P. Biensan, P. Kasztejna, K. Nechev, and R. J. Staniewicz, Journal of Power Sources, 97-98, 13 (2001).

29. S.-P. Kim, A. C. T. v. Duin, and V. B. Shenoy, Journal of Power Sources, 196, 8590 (2011).

30. J. Feng, C. Liu, J. Shen, and P. Yue, in Modeling of Soft Matter, M.-C. Calderer and E. Terentjev Editors, p. 1, Springer New York (2005).

31. J. Yan, B.-J. Xia, Y.-C. Su, X.-Z. Zhou, J. Zhang, and X.-G. Zhang, Electrochimica Acta, 53, 7069 (2008).

32. K. Edström, M. Herstedt, and D. P. Abraham, Journal of Power Sources, 153, 380 (2006).

33. E. Peled, D. Golodnitsky, and G. Ardel, Journal of the Electrochemical Society, 144, L208 (1997).

34. C. V. Di Leo, E. Rejovitzky, and L. Anand, Journal of the Mechanics and Physics of Solids, 70, 1 (2014).

35. G. Sarre, P. Blanchard, and M. Broussely, Journal of Power Sources, 127, 65 (2004).

36. L. Liu and M. Zhu, ECS Transactions, 61, 43 (2014).

37. J. Christensen and J. Newman, Journal of the Electrochemical Society, 151, A1977 (2004).

38. L. Liu, J. Park, X. Lin, A. M. Sastry, and W. Lu, Journal of Power Sources, 268, 482 (2014).

39. P. Guan, X. Lin, and L. Liu, ECS Transactions, 61, 29 (2014).

40. M. Ode, S. G. Kim, and T. Suzuki, ISIJ International, 41, 1076 (2001)

41. L.-Q. Chen, Annual Review of Materials Research, 32, 113 (2002)

42. G. J. W. Jie Deng and Richard P. Muller, Journal of the Electrochemical Society, 160, A487 (2013).

43. A. Powell and W. Pongsaksawad, Simulation of Electrochemical Processes II, 54, 43 (2007).

44. W. Gathright, M. Jensen, and D. Lewis, Electrochemistry Communications, 13, 520 (2011).

45. J. E. Guyer, W. J. Boettinger, J. A. Warren, and G. B. McFadden, Physical Review E, 69, $12(2004)$

46. J. E. Guyer, W. J. Boettinger, J. A. Warren, and G. B. McFadden, Physical Review E, 69, 13 (2004).

47. B. C. Han, A. Van der Ven, D. Morgan, and G. Ceder, Electrochimica Acta, 49, 4691 (2004).

48. Y. Shibuta, Y. Okajima, and T. Suzuki, Science and Technology of Advanced Materials, 8, 511 (2007)

49. W. Gathright, M. Jensen, and D. Lewis, in Solid Oxide Fuel Cells 12, S. C. Singhaland K. Eguchi Editors, p. 1077, ElectrochemicalSocietyInc, Pennington (2011).

50. P. Yue, J. J. Feng, C. Liu, and J. Shen, J. Fluid Mech., 515, 297 (2004).

51. C. Z. P. Yue and J. J. Feng, Journal of Computational Physics, 219, 47 (2006) 
52. P. Guan and L. Liu, MRS Proceedings, 1753 (2015).

53. P. Yue, J. J. Feng, C. Liu, and J. Shen, Journal of Fluid Mechanics, 515, 293 (2004).

54. P. Yue, C. Zhou, and J. J. Feng, Journal of Computational Physics, 223, 1 (2007).

55. D. J. Trtton, Physical Fluid Dynamisc, 2nd ed., Oxford University Press (1988).

56. T. Nishida, K. Nishikawa, and Y. Fukunaka, ECS Transactions, 6, 1 (2008).

57. K. Tasaki, A. Goldberg, J.-J. Lian, M. Walker, A. Timmons, and S. J. Harris, The Electrochemical Society, 156, A1019 (2009).
58. K. Persson, V. A. Sethuraman, L. J. Hardwick, Y. Hinuma, Y. S. Meng, A. van der Ven, V. Srinivasan, R. Kostecki, and G. Ceder, The Journal of Physical Chemistry Letters, 1, 1176 (2010).

59. M. B. Pinson and M. Z. Bazant, J. Electrochem. Soc., 160, A243 (2013).

60. A. V. Churikov, A. V. Ivanishchev, A. V. Ushakov, and V. O. Romanova, J Solid State Electrochem, 18, 1425 (2014)

61. V. Lesch, S. Jeremias, A. Moretti, S. Passerini, A. Heuer, and O. Borodin, The Journal of Physical Chemistry B, 118, 7367 (2014). 